\title{
Análisis de las Sentencias Registradas Judicialmente sobre Delitos de Violencia Intrafamiliar e Impacto de la Aplicación de la Ley Orgánica 1/2004
}

\section{Analysis of Court Sentences on Crimes of Domestic Violence and Impact of Implementation of Organic Law 1/2004}

\author{
Cesar San Juan Guillén y Anabel Vergara Iraeta
}

Universidad del País Vasco

\begin{abstract}
Resumen. Se presentan los resultados de una investigación en la que se analizan las sentencias de los juzgados de violencia doméstica del País Vasco. El periodo de estudio abarca desde el año 2004, hasta diciembre de 2007, es decir, cuatro años que, por otra parte, coinciden con el inicio de la aplicación de la LEY ORGÁNICA 1/2004, de 28 de diciembre, de Medidas de Protección Integral contra la Violencia de Género. De esta forma, el trabajo presentado ofrece algunos indicadores de evaluación de dicha ley, tal como la evolución de la incidencia de casos y la reincidencia. También se ofrecen resultados descriptivos sobre el perfil de la víctima y el imputado en las diferentes formas de violencia intrafamiliar registradas. Se discuten los retos planteados para resolver judicial y penalmente este problema psicosocial, así como las dificultades para establecer criterios de eficacia de las leyes que sancionan los actos de violencia producidos en el ámbito doméstico.
\end{abstract}

Palabras clave: violencia intrafamiliar, género, LO 1/2004, sentencias, reincidencia.

\begin{abstract}
We show the results of a study which analyzes the sentences of Domestic Violence Courts in the Basque Country. The period of study covers the years from 2004 to 2007. Four years that, on the other hand, are overlapped with the beginning of the ORGANIC LAW 1/2004 application, of Integral Protection Measures Against the Genre Violence. On this way, this work offers some indicators of evaluation about mentioned law, such as the incidence evolution of cases and the recidivism. Descriptive results about the profile of the victim and the imputed in the different forms of domestic violence registered are also offered. Challenges raised to resolve judicially this psychosocial problem and the difficulties to establish efficiency criteria of the laws that sanction the acts of violence produced in the domestic area are discussed
\end{abstract}

Keywords: domestic violence, genre, LO 1/2004, judgments.

\section{Introducción}

La puesta en práctica de la LEY ORGÁNICA 1/2004, de 28 de diciembre, de Medidas de Protec-

La correspondencia sobre este artículo debe enviarse al primer autor al e-mail: cesar.sanjuan@ehu.es ción Integral contra la Violencia de Género constituye una de las herramientas de las que se ha dotado el Estado y, en última instancia, la sociedad para intentar reducir la incidencia de un problema complejo como es el de la violencia intrafamiliar, en general, y la violencia contra las mujeres, en particular. Sin embargo, las estadísticas no nos hacen ser 
muy optimistas a la hora de evaluar la eficacia de dicho instrumento legal ya que todo parece indicar que el fenómeno no muestra indicios significativos de recesión. Según datos del Departamento de Justicia del Gobierno Vasco, cada día se presentan en esta comunidad once denuncias por violencia machista. Según esta misma fuente, los asuntos penales por violencia de género se han incrementado en un $10 \%$ entre 2005 y 2007, pasando de 3.761 a 4.127. Por su parte, las órdenes de protección solicitadas han crecido un 50\%. En total, el $67 \%$ de los denunciados son condenados y el $33 \%$ absueltos. Cabe destacar, por otra parte, que el $25 \%$ de esas denuncias son interpuestas por mujeres extranjeras. Si tenemos en cuenta que la población general del País Vasco tiene aproximadamente un 5\% de personas extranjeras, nos puede dar una idea de un primer esbozo del problema. Todas estas cifras en aumento,- aunque sea discutible si realmente son un indicador de que la sociedad está cada vez más sensibilizada y en consecuencia se denuncia más o, por el contrario, reflejan un incremento objetivo de la incidencia del problema,- al margen de cómo las interpretemos, nos sitúan ante un problema dramático y vergonzoso para una sociedad moderna y pretendidamente avanzada.

De todas formas, tratándose como hemos dicho anteriormente de un problema complejo, sería muy ingenuo que esperemos resolverlo únicamente en el terreno penal aunque constituye, sin duda, una perspectiva necesaria. No sería suficiente porque, entre otras razones, no se puede ocultar la posibilidad de que se pueda estar produciendo una implementación de las herramientas legales poco eficiente, en el sentido de que existan mujeres, ancianos y menores maltratados en el ámbito familiar que van a ser invisibles para el sistema judicial. Un déficit de eficiencia del sistema que podría llegar a la perversión si se confirmara la existencia de supuestas denuncias falsas o, cuando menos, digámoslo así, se esté produciendo una excesiva judicialización de los conflictos familiares.

Desde este punto de vista, nos parece necesario hacer mayores esfuerzos por conocer las causas de la violencia intrafamiliar y sus consecuencias, pero también por conocer, con la mayor exactitud posible, todas las circunstancias que rodean la pluralidad de agresiones que se pueden ejercer en el contexto familiar. De esta inquietud es como surge la necesidad de desarrollar un estudio de las características que aquí se presenta, en el que se muestra el análisis realizado a partir de las sentencias condenatorias dictadas en los juzgados de violencia doméstica de Vizcaya, Álava y Guipúzcoa. Se trata de un estudio exploratorio, de carácter descriptivo, que ha tenido como principal limitación la gestión de unos datos provenientes de bases separadas e inconexas. Los objetivos son, por tanto, prudentes y centrados en obtener información básica relativa a las características de la delincuencia cometida en el ámbito doméstico y entre las relaciones familiares, a su evolución en el periodo de tiempo estudiado (20042007), a las tasas de reincidencia y a características de las víctimas, tales como su nacionalidad, la relación de parentesco con sus agresores o la edad.

\section{Conceptos en violencia intrafamiliar}

Existe entre la opinión pública, la Administración, las organizaciones sociales, e incluso entre las referencias científicas especializadas, un amplio catálogo de conceptos para referirse a los actos violentos y otras conductas constitutivas de delito cometidos en el ámbito familiar o de las relaciones afectivas. Desde la, a nuestro modo de ver, agotada etiqueta de "violencia de género" hasta la muy imprecisa "violencia doméstica" pasando por la discutible "violencia machista", se vindica dicha nomenclatura para, desde sus matices semánticos, subrayar o poner el acento sobre la asimetría de las relaciones, el escenario de conducta o el rol criminógeno masculino, respectivamente. En la línea de investigación que venimos desarrollando en el Instituto Vasco de Criminología hemos tomado la decisión de referirnos a este ámbito de estudio como violencia intrafamiliar ya que, al margen de cómo se defina la "familia", los hechos que se analizan se producen en un contexto que es reconocido como "familiar" por los protagonistas de los mismos. Desde este punto de vista, cuando hablamos de violencia intrafamiliar, nos estamos refiriendo a todas las acciones de maltrato (psicológico, físico, sexual), comportamiento negligente, abuso de po- 
der, etc., de un miembro de la familia sobre otro o que, en todo caso, se desarrollan en el contexto de las relaciones familiares o afectivas y que pueden ocasionar diversos niveles de daño a las víctimas.

Por otra parte, al hablar de las víctimas nos estamos refiriendo fundamentalmente a las mujeres, los menores y a las personas de avanzada edad o personas dependientes. Sin embargo, siendo éstas las principales formas de relación agresiva o negligente, también nos podemos encontrar con procesos judiciales entre hermanos o cualquier otro vector de parentesco hasta el punto de encontrarnos con familias en las que se ha instalado una dinámica de violencia doméstica (Ballonga et al, 2007). Los tres supuestos básicos referidos tienen una serie de elementos comunes pero también unas características diferenciales que describiremos sucintamente a continuación. Las estadísticas mostradas en este apartado están obtenidas del Instituto de la Mujer (http://www.migualdad.es/mujer/), el Ministerio del Interior (http://www.mir.es/) y el Centro Reina Sofía para el estudio de la Violencia (http://www.centroreinasofia.es/). Todas las figuras son de elaboración propia.

\section{Violencia contra la mujer}

La violencia contra las mujeres constituye una de las modalidades más frecuentes y relevantes entre las diferentes formas de violencia intrafamiliar. Es una forma de relación de abuso entre quienes sostienen o han sostenido un vínculo afectivo relativamente estable, incluyendo relaciones de matrimo- nio, noviazgo, pareja (con o sin convivencia). Se enmarca en un contexto de desequilibrio de poder e implica un conjunto de acciones, conductas y actitudes que se mantienen como estilo relacional y de interacción imperante en la pareja donde normalmente un hombre, por acción u omisión, ocasiona daño físico y/o psicológico a una mujer.

Las cifras que hemos adelantado y las que ofreceremos en el presente estudio, explican y justifican los esfuerzos e iniciativas que apuntan a la mujer como víctima principal. Desde un punto de vista psico-ambiental, el espacio de mayor riesgo de una mujer para sufrir violencia es su propio hogar, al contrario de lo que sucede a los hombres, para quienes el espacio de mayor riesgo es la calle.

La violencia sobre la mujer puede tomar muchas formas, desde las más sutiles y difíciles de diferenciar hasta las más brutales. Puede ocurrir en cualquier etapa de su vida, incluyendo el embarazo y puede afectar tanto desde un punto de vista físico como mental.

Podemos observar en el Gráfica 1 la evolución en España de este desazonante protagonismo de la mujer maltratada en el ámbito familiar. La inversión de tendencia, en lo que concierne a las Faltas a partir del año 2004, se debe precisamente a la implementación de la citada Ley Integral que, en el fondo, ha significado un endurecimiento de las clasificaciones penales. En todo caso, las cifras absolutas, sumando delitos y faltas, reflejan un crecimiento sostenido en el que, el punto de inflexión que pudiera haber supuesto el endurecimiento de las penas, no ha tenido el esperado efecto disuasorio. En todo caso habría que contrastar este extremo con la evo-

Gráfica 1. Incidencia de mujeres maltratadas en el ámbito familiar entre 2002 y 2006 (Aumento del 41,75\%)

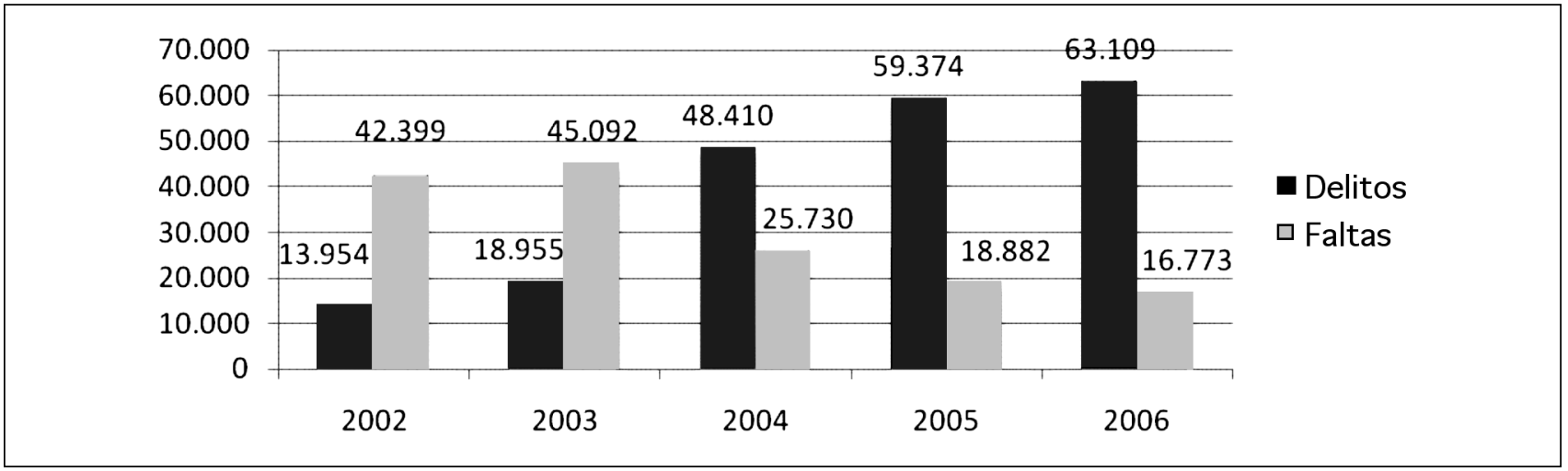


lución de la población total para confirmar si existe un crecimiento en términos relativos.

Exactamente la misma tendencia observamos en el Gráfica 2 referida a las mujeres maltratadas por su pareja. por las consecuencias inmediatas, a mediano $\mathrm{y}$ largo plazo que generan en el desarrollo del menor.

Dentro de esta categoría podemos clasificar también el abuso fetal que ocurre cuando la futura

Gráfica 2. Incidencia de mujeres maltratadas por su pareja entre 2002 y 2006 (Aumento del 43,53\%)

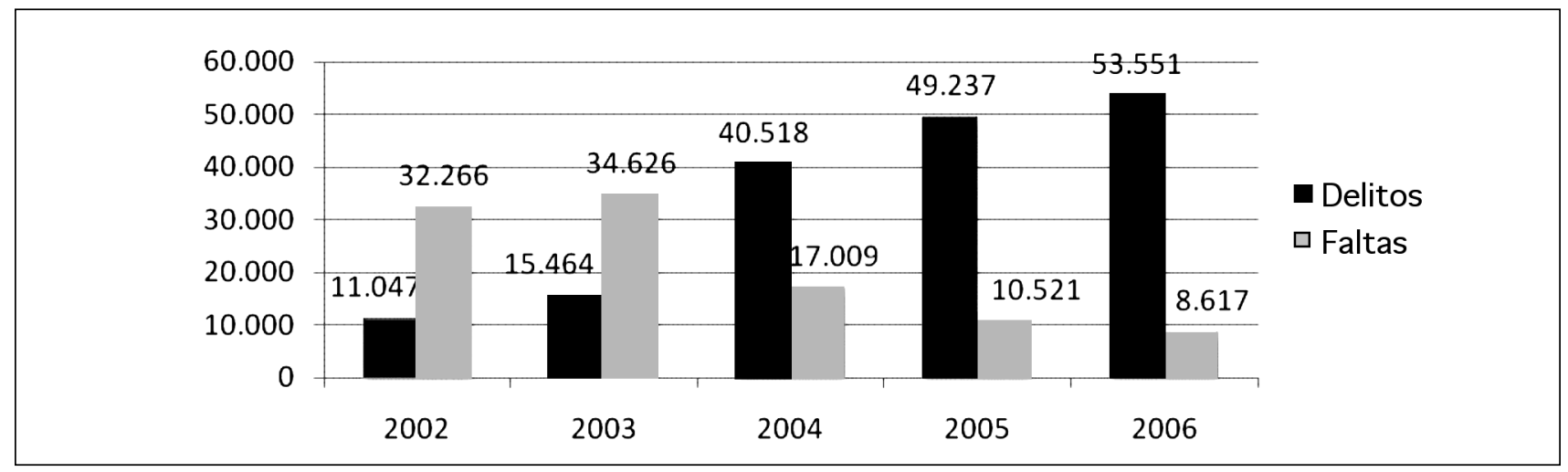

\section{Maltrato infantil.}

El maltrato infantil, de manera general, puede definirse como todo acto no accidental, único o repetido que por acción u omisión (falta de la respuesta o acción apropiada) provoca daño físico o psicológico a una persona menor de edad, ya sea por parte de sus padres, otros miembros de la familia o cuidadores que, aunque externos a la familia, deben ser supervisados por ésta. El maltrato infantil incluye el abandono completo o parcial y todo comportamiento o discurso adulto que infrinja o interfiera con los Derechos del Niño. La violencia, ya sea física, sexual o emocional es una de las más graves infracciones a estos derechos, madre ingiere, deliberadamente, alcohol o drogas estando el feto en su vientre, pudiendo el niño o niña, en consecuencia, nacer con adicciones, malformaciones o retraso severo, entre otros problemas. Los expertos calculan que sólo un 10 ó un $20 \%$ de los casos de maltrato infantil sale a la luz. El miedo, la sensación de culpabilidad y su corta edad inciden en su falta de visibilidad. De hecho, podríamos decir que el maltrato infantil y, aún en mayor medida, el maltrato a los ancianos tienen el mayor índice de invisibilidad de las formas de violencia intrafamiliar. De los casos constatables, podemos observar en el Gráfica 3 una tendencia al alza que confirma lo preocupante de este fenómeno.

Gráfica 3. Incidencia de menores maltratados en el ámbito familiar entre 2001 y 2005. Ha aumentado un 146,29\%

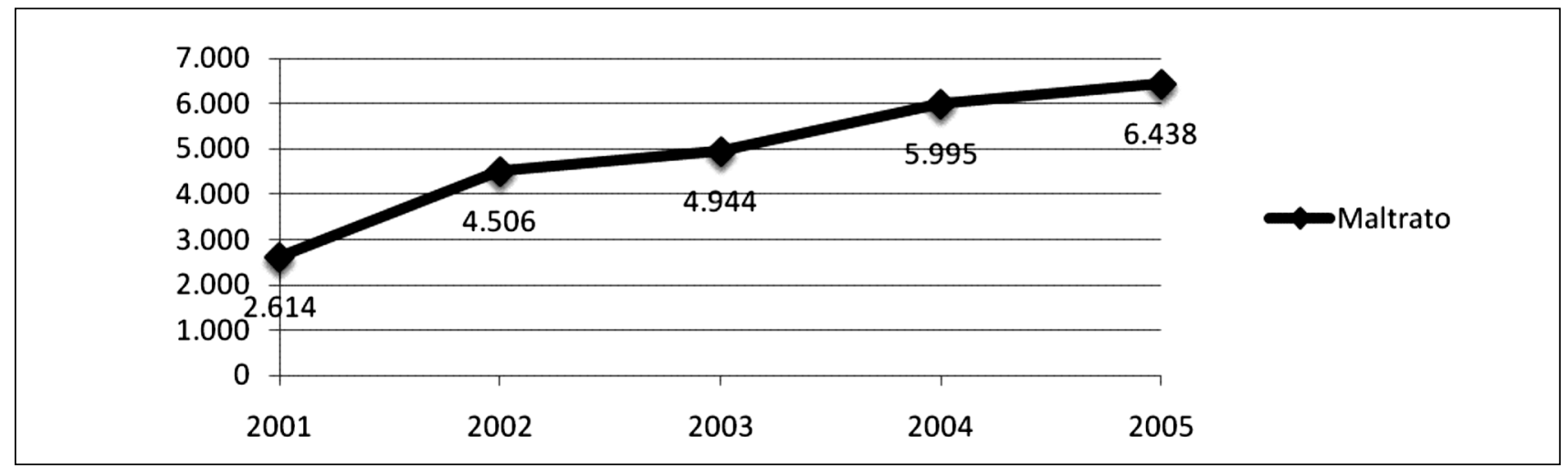




\section{Violencia contra las personas mayores.}

La violencia o el maltrato a los ancianos y ancianas puede definirse como todo acto no accidental, único o repetido, que por acción u omisión (falta de la respuesta o acción apropiada) provoca daño físico o psicológico a una persona anciana, ya sea por parte de sus hijos, otros miembros de la familia o de cuidadores. Estas situaciones de maltrato son una causa importante de lesiones, enfermedades, pérdida de interés, aislamiento y desesperación. Uno de los factores que explican el maltrato hacia las personas ancianas tiene que ver con nuestra propia cultura en la que se ha consolidado una representación social de la ancianidad próxima a lo inservible e inútil. Las personas ancianas son percibidas como estorbos o como una carga, por eso no es de extrañar que el tipo más frecuente de maltrato sea el abandono y la falta de cuidados.

Por otra parte, la ausencia de registros o estimaciones reales de la dimensión de este problema, así como la escasez de denuncias debido al miedo, la depresión, la incapacidad de moverse por sí mismos y la poca credibilidad, ha permitido que este fenómeno sea, como hemos mencionado anteriormente, prácticamente invisible (de la Cuesta, 2006). A pesar de esta falta de visibilidad la incidencia de los casos detectados también es creciente, como se aprecia en la Gráfica 4.

\section{Análisis de las sentencias dictadas por los juzga- dos de Violencia Doméstica del País Vasco en el periodo 2004-2007}

\section{Procedimiento}

El objetivo de este estudio es realizar un análisis descriptivo de las sentencias dictadas por los juzga- dos de Violencia Doméstica del País Vasco. El periodo de estudio abarca desde el año 2004 inclusive, hasta diciembre de 2007, es decir, prácticamente cuatro años que, por otra parte, corresponde al periodo de aplicación de la LEY ORGÁNICA 1/2004, de 28 de diciembre, de Medidas de Protección Integral contra la Violencia de Género. El aspecto que más ha podido dificultar el proceso de análisis de las sentencias es el hecho de que no hemos dispuesto de una base unificada de datos, como hubiera sido deseable. Por el contrario, se ha trabajado con 18 matrices distintas correspondientes a cada uno de los elementos del proceso penal objeto de análisis, es decir: Causas, Denunciantes, Imputados, Delitos, Medidas y Condenas y estos, a su vez, distribuidos en tres bases diferentes correspondientes a los juzgados de Álava, Vizcaya y Guipúzcoa. A todo esto, debemos añadir que, en contra de lo que suele ser habitual en una matriz de datos en la que cada fila de la base objeto de análisis es un individuo, en el caso que nos ocupa, algunas matrices de datos presentan cada fila como una "Causa" esto es, un procedimiento penal, lo que en ocasiones nos va a obligar a "pensar" los resultados en función de esta unidad de análisis, además de no poderla relacionar con aquellas en las que cada fila es un sujeto. Dicha situación limita enormemente las posibilidades de extraer conclusiones más allá de las puramente descriptivas.

\section{Resultados}

\section{Formas de violencia intrafamiliar}

En la introducción hemos hecho referencia a los tipos más frecuentes de violencia familiar en función de la relación o parentesco entre víctima y agre-

Gráfica 4. Incidencia de personas ancianas maltratadas en el ámbito familiar (Aumento del un 82,27\%)

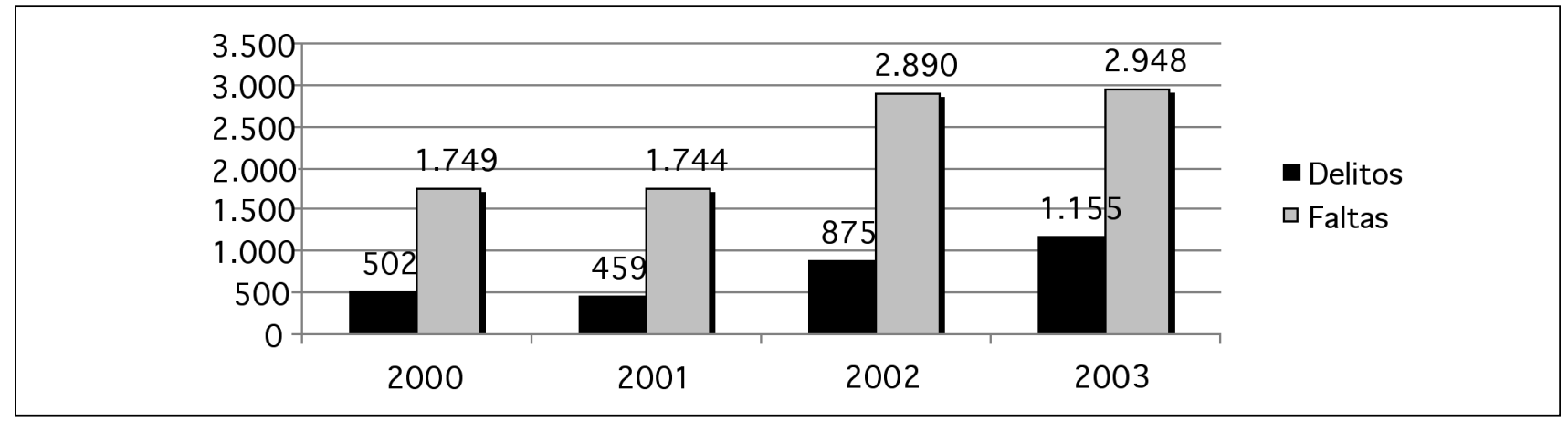


sor. Desde un punto de vista victimológico, hemos destacado la importancia de las mujeres, los menores y las personas ancianas.

En el caso que nos ocupa, podemos observar en la tabla 1, las diferentes formas de violencia familiar de la muestra estudiada teniendo en cuenta que, como ya hemos advertido, los indicadores de frecuencia corresponden a causas, no a individuos, por lo que existen más relaciones de parentesco que individuos encausados. Por ello sugerimos atender a los porcentajes, ya que pueden reflejar más adecuadamente la proporción en la que se manifiesta cada tipo de parentesco.

Asimismo, los resultados indican que en algo más de la mitad de los casos (58\%), no existe convivencia entre ambas partes, lo que también nos da una idea de que el problema de la violencia traspasa en muchas ocasiones el ámbito puramente "doméstico". Hemos observado en la tabla 1 una relación muy exhaustiva de tipos de relaciones familiares y afectivas en la que, en primer lugar, podemos prestar atención al peso específico que tiene la violencia contra la mujer en la definición y análisis del problema de la violencia intrafamiliar. Quizás este extremo pueda apreciarse más claramente en la agrupación que hemos realizado en la Gráfica 5. De hecho, incluso asumiendo la existencia de un porcentaje poco significativo de violencia contra el hombre en

Tabla 1. Frecuencia de tipos de relación entre víctima e imputado

\begin{tabular}{lrr}
\hline Parentesco & Frecuencia & Porcentaje \\
\hline Abuelo/a & 15 &, 3 \\
Cónyuge & 1968 & 33,0 \\
Ex-pareja de hecho & 703 & 11,8 \\
Ex-cónyuge & 434 & 7,3 \\
Ex-novio & 595 & 10,0 \\
Hijo/a del cónyuge & 40 &, 7 \\
Hermano/a & 195 & 3,3 \\
Hijo/a de la pareja de hecho & 11 &, 2 \\
Hijo/a & 198 & 3,3 \\
Incapaz sujeto a tutela & 3 &, 1 \\
Madre & 236 & 4,0 \\
Menor que convive & 6 &, 1 \\
Menor sujeto a potestad & 94 & 1,6 \\
Nieto/a & 7 &, 1 \\
Novio/a & 246 & 4,1 \\
Otro tipo & 321 & 5,4 \\
Padre & 63 & 1,1 \\
Pareja de hecho & 793 & 13,3 \\
En proceso de separación & 43 &, 7 \\
\hline Total & 5971 & 100,0 \\
\hline
\end{tabular}

el seno de la pareja, podemos decir que la violencia contra la mujer constituye aproximadamente el $80 \%$ del fenómeno de la violencia intrafamiliar, al menos en lo que concierne a lo que resulta visible para el sistema penal.

Si tomáramos como referencia esta submuestra de casos de violencia en el seno de las relaciones de pareja, vemos que existen algunas diferencias si la comparamos con los casos registrados en el resto de España (Gráfica 6). Hay que tener en cuenta que, en el estudio que hemos realizado en País Vasco, la unidad de análisis es la "causa penal" y en el caso de España la unidad de análisis son las víctimas (Fuente: Consejo General del Poder Judicial). Aún así, en el caso del País Vasco, parece existir una especial incidencia de casos de violencia entre cónyuges.

\section{Gravedad de los delitos}

La casuística de delitos cometidos sobrepasa, jurídicamente hablando, las formas de violencia que hemos descrito en la introducción (como por ejemplo, delitos contra la Administración de Justicia), o son delitos "colaterales" al daño infringido a la víctima (como por ejemplo la conducción alcohólica). En este sentido, también podemos diferenciar diversos niveles de gravedad en cuanto al daño producido. Por eso es necesario realizar un análisis de esta variable en los delitos registrados, con el fin de aportar un nuevo elemento de concreción a la definición del problema. Para ello, hemos establecido el siguiente criterio, consistente en una escala de gravedad de 5 niveles, que vamos a tomar como referencia para re-categorizar el catálogo de delitos identificados en la muestra de sentencias estudiadas:

- Gravedad extrema: resultados irreversibles que afecten a la vida humana.

- Muy grave: injerencias significativas en la integridad corporal o salud psicofísica con uso de medios especialmente deletéreos o causación de resultados significativos desde el prisma de la indemnidad física o la salud psicofísica; lesión de la libertad sexual a través de la violencia o la intimidación.

- Grave: injerencias relevantes en la integridad 
Gráfica 5. Tipo de relación entre víctima e imputado en la Muestra del País Vasco

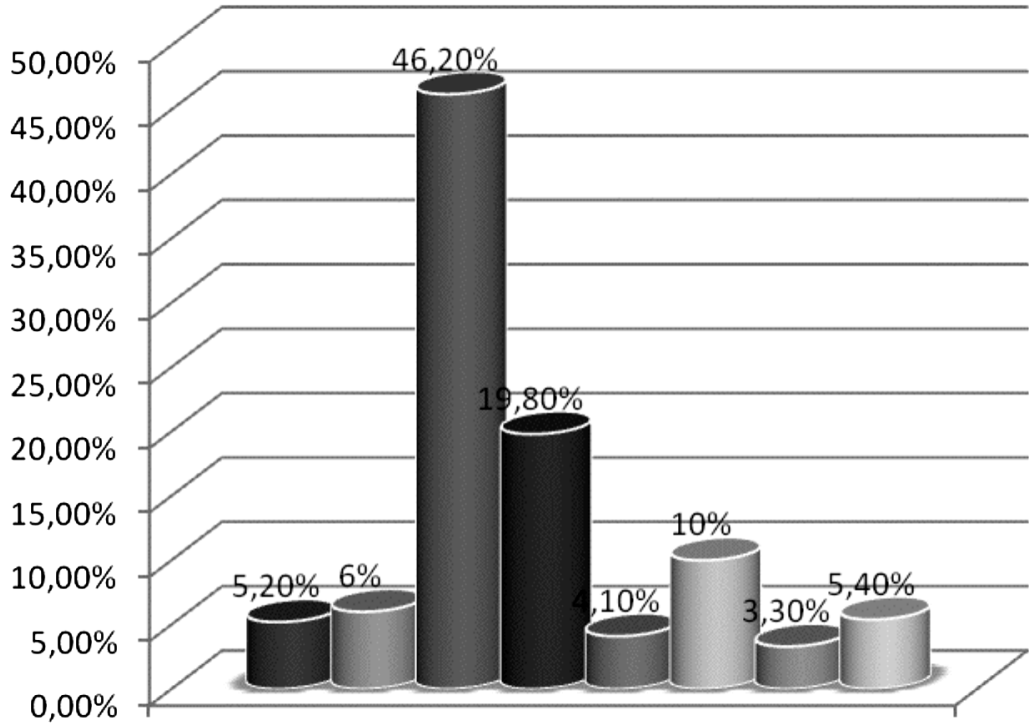

Parentesco
- Contra ascendente

- Contra descendiente

- Cónyuges o Pareja

- ExCónyuges

- Relac. Afectiva

ExRelac. Afectiva

- Hermanos

Otros

Gráfica 6. Tipo de relación en los casos de violencia en la pareja. (Comparativa País Vasco-España)

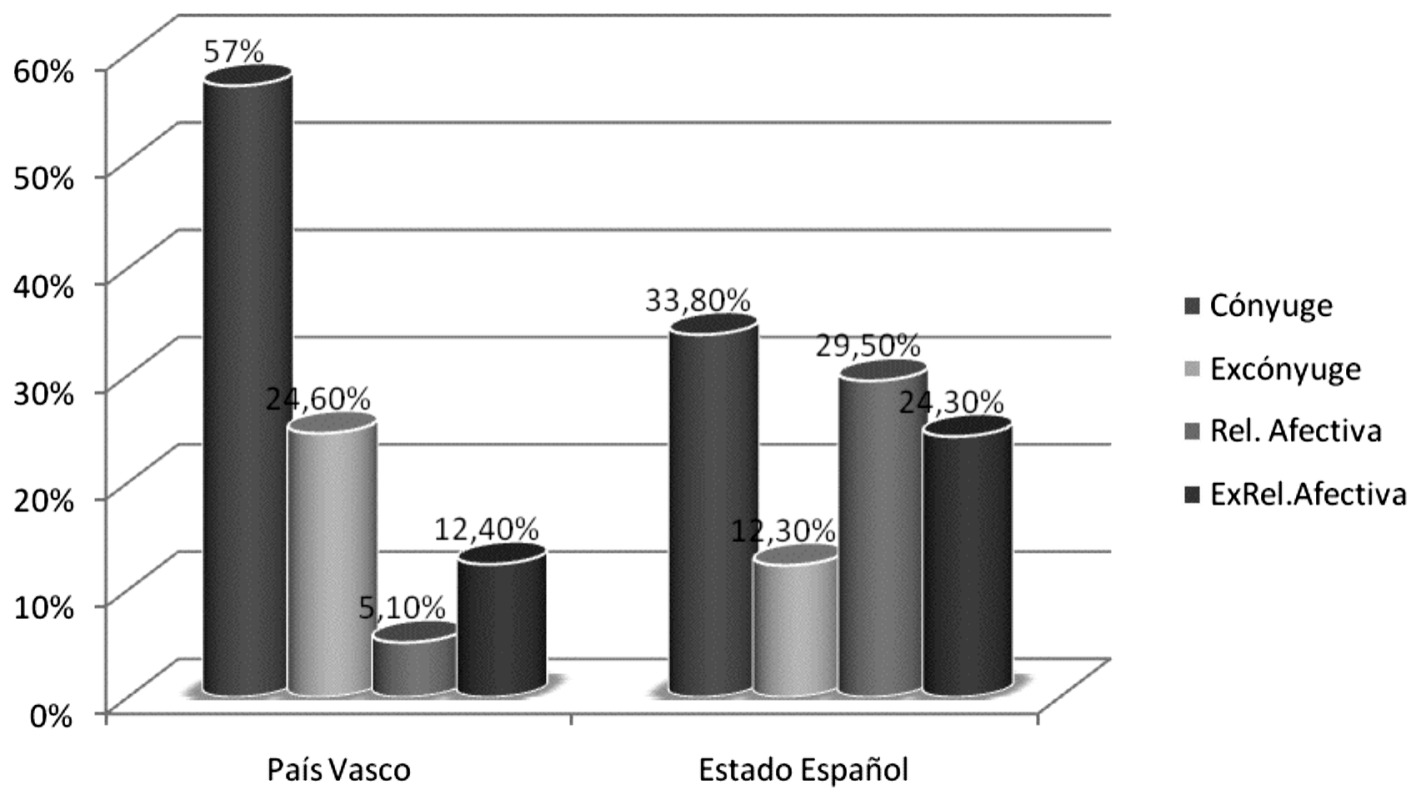

corporal o salud psicofísica, creación de un espacio de dominio psicofísico en el contexto familiar, afectaciones de la libertad personal, lesión de la libertad sexual, empleo de la violencia o la intimidación para lesionar el patrimonio ajeno.
- Moderado: lesiones no significativas en el contexto familiar, injerencias significativas en la integridad corporal o salud psicofísica por imprudencia grave, participación en un contexto idóneo para afectar a la vida o causar significativas afecciones de la integridad corporal o la 
salud psicofísica, involucrar a otra persona en un contexto sexual no voluntario, omitir la ayuda en una situación de peligro para bienes personales de otro, invadir la privacidad ajena en un contexto tan sensible para la percepción de intimidad y seguridad personal como el domicilio, afectar a la dignidad ajena con expresiones o palabras, emplear medios específicos para quebrar el marco de custodia del patrimonio ajeno, causar desperfectos relevantes en el patrimonio ajeno de forma intencionada, producir daños colectivos de forma imprudente, crear un riesgo específico para la vida y salud ajena en un contexto de riesgo significativo como la circulación de vehículos a motor y afectar de forma relevante a los intereses públicos o institucionales.

- Leve: las infracciones constitutivas de falta.

Dada la representatividad del juzgado de Violencia Doméstica de Bizkaia (si atendemos a su nivel de actividad) para este primer análisis hemos seleccionado las sentencias registradas en el mismo. Como podemos apreciar en la tabla 2, los porcentajes correspondientes a los delitos тиу graves o extremadamente graves tienen una baja incidencia. Aunque tratándose de delitos que atentan gravemente contra la integridad física de la víctima, llegando al asesinato en el nivel máximo de la escala de gravedad, debemos defender que siempre nos van a resultar cifras extraordinariamente altas.

Tabla 2. Gravedad de los delitos cometidos registrados en el Juzgado de VD de Bizkaia

\begin{tabular}{lc}
\hline Gravedad & Porcentaje \\
\hline Leve & $18 \%$ \\
Moderado & $61,2 \%$ \\
Grave & $19,7 \%$ \\
Muy Grave & $0,75 \%$ \\
Extrema & $0,3 \%$ \\
\hline
\end{tabular}

En esta línea, el Grupo de expertos en violencia Doméstica y de Género del Consejo General del Poder Judicial, realiza un interesante análisis centrándose en los casos de asesinato. En dicho estudio, tras analizar 147 sentencias sobre asesinatos machistas, dictadas por los tribunales del jurado entre 2001 y 2005 concluye que el alcohol, las drogas o las alteraciones psíquicas sólo han sido atenuante del delito en el 3,4\% y el 5,4\% de los casos, respectivamente. Ello permite cuestionar algunas falsas creencias muy generalizadas y enraizadas en la conciencia social, según se sostiene en el informe. El retrato que se describe corresponde a un varón, extremadamente violento, de entre 30 y 45 años que actúa con premeditación y plena consciencia. En una cuarta parte de las sentencias analizadas, la relación entre el homicida y su víctima ya había terminado. Por nacionalidades, el 26,9\% de los acusados eran extranjeros, encontrándose esta misma proporción entre las víctimas. En dicho informe se hace referencia a esta sobre-representación de víctimas extranjeras, ya que se trata de cifras más elevadas que la proporción de extranjeros con respecto a la población general española, en la fecha estudiada $(8,46 \%$ en 2005).

\section{Evolución de la incidencia de sentencias dictadas}

El volumen de trabajo de los juzgados de violencia doméstica puede ser indicador de la magnitud del problema. ¿Es, por tanto, una evolución descendente de la incidencia de casos registrados judicialmente lo que vamos a considerar un logro de los planes de prevención e intervención?. Este hecho, sin embargo, podría colisionar con otros objetivos de políticas sociales centradas en incentivar la sensibilidad social en lo que concierne a dar el paso de denunciar al maltratador... ¿Esperamos entonces una evolución ascendente de la incidencia en los juzgados como indicador de éxito de dichas iniciativas publicitarias?

También, claro está, este último resultado puede ser un indicador de eficacia en el funcionamiento de unos juzgados, de los que se espera prontitud en la respuesta de protección a la víctima y sanción al infractor.

Es decir, en función de cuáles sean las intenciones de quien hace la propuesta, las interpretaciones de las memorias judiciales pueden orientarse en una $\mathrm{u}$ otra dirección. A nosotros este ejercicio nos resulta científicamente baladí y, a falta de otros elementos de juicio, consideramos arriesgado sugerir conclu- 
Gráfica7. Evolución de la incidencia de sentencias dictadas durante 2004-2007.

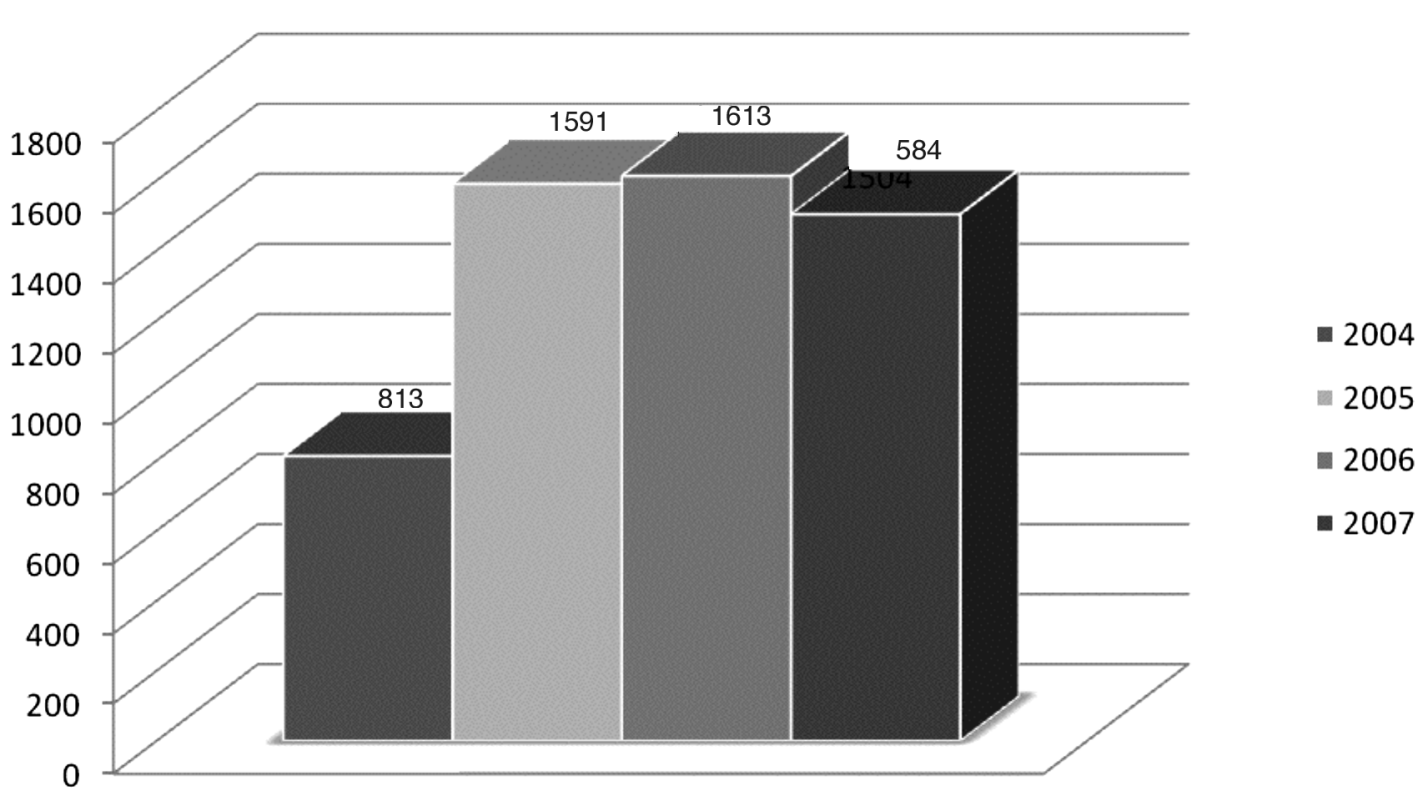

siones que pudieran ser precipitadas. En lo que concierne a nuestros datos, si observamos la Gráfica 7, cabría destacar la línea más o menos sostenida de los años 2005, 2006 y 2007. El cambio de tendencia es sin duda llamativo en el año previo a este trienio. Del año 2004 a los años subsiguientes prácticamente se dobla el número de sentencias dictadas, lo que requeriría una reflexión en profundidad sobre el impacto de la Ley Orgánica 1/2004.

\section{Nacionalidad de imputados y denunciantes}

Otra variable que, desde un punto de vista psi-

\begin{tabular}{lrr}
\hline \multicolumn{3}{c}{ Tabla 3. Nacionalidad de los imputados } \\
\hline Nacionalidad & Frecuencia & Porcentaje \\
\hline Española & 4.470 & $76,8 \%$ \\
Ecuatoriana & 234 & $4 \%$ \\
Colombiana & 176 & $3 \%$ \\
Boliviana & 165 & $2,8 \%$ \\
Resto de América Latina & 177 & $3.1 \%$ \\
Marroquí & 127 & $2,2 \%$ \\
Resto de África & 109 & $1,9 \%$ \\
Portuguesa & 106 & $1,8 \%$ \\
Europa del Este & 93 & $1,6 \%$ \\
Resto Europa Occidental & 43 & $0,7 \%$ \\
Resto del Mundo, apátridas o NC & 121 & $2,1 \%$ \\
\hline
\end{tabular}

cosocial y criminológico, tiene un cierto interés a la hora de definir los perfiles de riesgo, es la nacionalidad, tanto de los imputados como de las víctimas.

Centrándonos en primer lugar en los imputados, hemos diseñado en la tabla 3 una relación de los colectivos más destacables en el que podrían hacerse alguna agrupaciones. En un análisis general de los resultados, cabe decir que un $76,8 \%$ de las sentencias son sobre imputados de nacionalidad española $\mathrm{y}$, por lo tanto, el porcentaje de sentencias sobre imputados extranjeros alcanzaría el 23,2\%. De este grupo, la mayor parte de las sentencias, corresponden a ecuatorianos, seguidos de colombianos y bolivianos. Por tanto, casi un $13 \%$ de las sentencias están relacionados con imputados latino-americanos.

Los casos relacionados con imputados de origen africano alcanzan un 4,1\%, siendo el marroquí, el colectivo más numeroso. El mismo porcentaje corresponde a casos relacionados con imputados europeos, donde los portugueses copan el $1,8 \%$ de las sentencias.

En lo que concierne a las víctimas, el perfil es algo diferente tal y como se puede apreciar en la tabla 4. En este caso, el porcentaje de causas relacionadas con víctimas de nacionalidad española 


\begin{tabular}{lcr}
\hline \multicolumn{3}{c}{ Tabla 4. Nacionalidad de los denunciantes } \\
\hline Nacionalidad & Frecuencia & Porcentaje \\
\hline Española & 5.361 & $78,9 \%$ \\
Colombiana & 245 & $3.6 \%$ \\
Boliviana & 201 & $3 \%$ \\
Ecuatoriana & 196 & $2,9 \%$ \\
Brasileña & 111 & $1,6 \%$ \\
Resto de América Latina & 151 & $2,2 \%$ \\
Marroquí & 55 & $0,8 \%$ \\
Resto de África & 74 & $1,1 \%$ \\
Portuguesa & 58 & $0,9 \%$ \\
Resto Europa Occidental & 19 & $0,3 \%$ \\
Rumana & 65 & $0,9 \%$ \\
Resto Europa del Este & 40 & $0,6 \%$ \\
Resto del Mundo o NC & 216 & $3,2 \%$ \\
\hline
\end{tabular}

alcanza el 78,9\%. Debemos interpretar con prudencia estas cifras ya que, en principio, pudiera pensarse, por poner el caso, que habiendo más imputados ecuatorianos que víctimas ecuatorias, aquellos tengan como víctimas a personas de otras nacionalidades. Sin embargo, debemos tener en cuenta que un mismo hecho puede ser denunciado por diversas personas, además de la propia víctima. Conviene atribuir a estos resultados, por tanto, un rango "orientativo". De todas formas, teniendo en cuenta que en el conjunto del Estado, y según datos del Consejo General del Poder Judicial, el $25 \%$ de las denunciantes de violencia machista son personas extranjeras, parece que no es imprudente otorgar cierta fiabilidad a nuestros resultados en los que el porcentaje de víctimas extranjeras podría rondar el $23 \%$, de las que, algo más de la mitad, serían latino-americanas. Todos estos resultados que hemos venido ofreciendo pueden suscitar algunas hipótesis, como por ejemplo la referida a si existe multi-reincidencia victimal, es decir, si una misma persona puede ser víctima de imputados diferentes, hipótesis que no podríamos contrastar con las características de las bases de datos disponibles. Por otra parte, si observamos la tabla 4 y la contrastamos con la tasa de población extranjera en el País Vasco, debe subrayarse algo que ya hemos comentado, y es la sobre-representación de población extranjera en los juzgados de violencia doméstica. Efectivamente, en el caso de la población general, (según datos del Observatorio Vasco de Inmigración en el año 2007) el mayor colectivo de extranjeros en la
Comunidad Autónoma Vasca, lo constituyen los colombianos, seguidos de los bolivianos; ranking que también vemos reflejado en la tabla 3 de víctimas. Lo que llama la atención es que, el tercer grupo de extranjeros en el País Vasco lo conforman el colectivo de rumanos y rumanas, entre los que no parecen existir tantos conflictos intrafamiliares y de pareja como en el caso de población latinoamericana.

En todo caso, nos parece oportuno apuntar una conclusión provisional acerca de la necesidad de prestar atencion a la alarmante prevalencia de un perfil sin duda en riesgo de exclusión social: la mujer inmigrante víctima.

\section{Edad de las víctimas}

Teniendo en cuenta lo que ya comentamos en la introducción acerca de los déficits de visibilidad social y judicial inherentes a la violencia contra menores y contra personas mayores, podemos observar el Gráfico 8, la curva en forma de "U" invertida en lo que respecta a la edad de los denunciantes de violencia intrafamilar. Acorde con otros estudios en los que se constata que la pertenencia a una franja de edad entre 30 y 40 años constituye uno de los "factores de riesgo" a la hora de definir el perfil de la víctima. A partir de este intervalo de edad vemos una reducción paulatina de los porcentajes entre los mayores de 40 años y los menores de 30. A medida que aumente la sensibilidad de la población hacia el problema del maltrato a menores y personas ancianas, sería interesante observar cómo evoluciona este fenómeno en los próximos años.

\section{Elementos de las causas: Medidas de protección a la víctima y demora en dictar sentencia}

Como podemos observar en la tabla 5 las medidas más habituales son la prohibición de aproximarse y la prohibición de comunicarse.

En párrafos anteriores planteábamos la discusión de considerar el volumen de sentencias dictadas de un juzgado como indicador de eficacia. De lo que no 
Gráfica 8. Edad de las víctimas

\section{Edad}

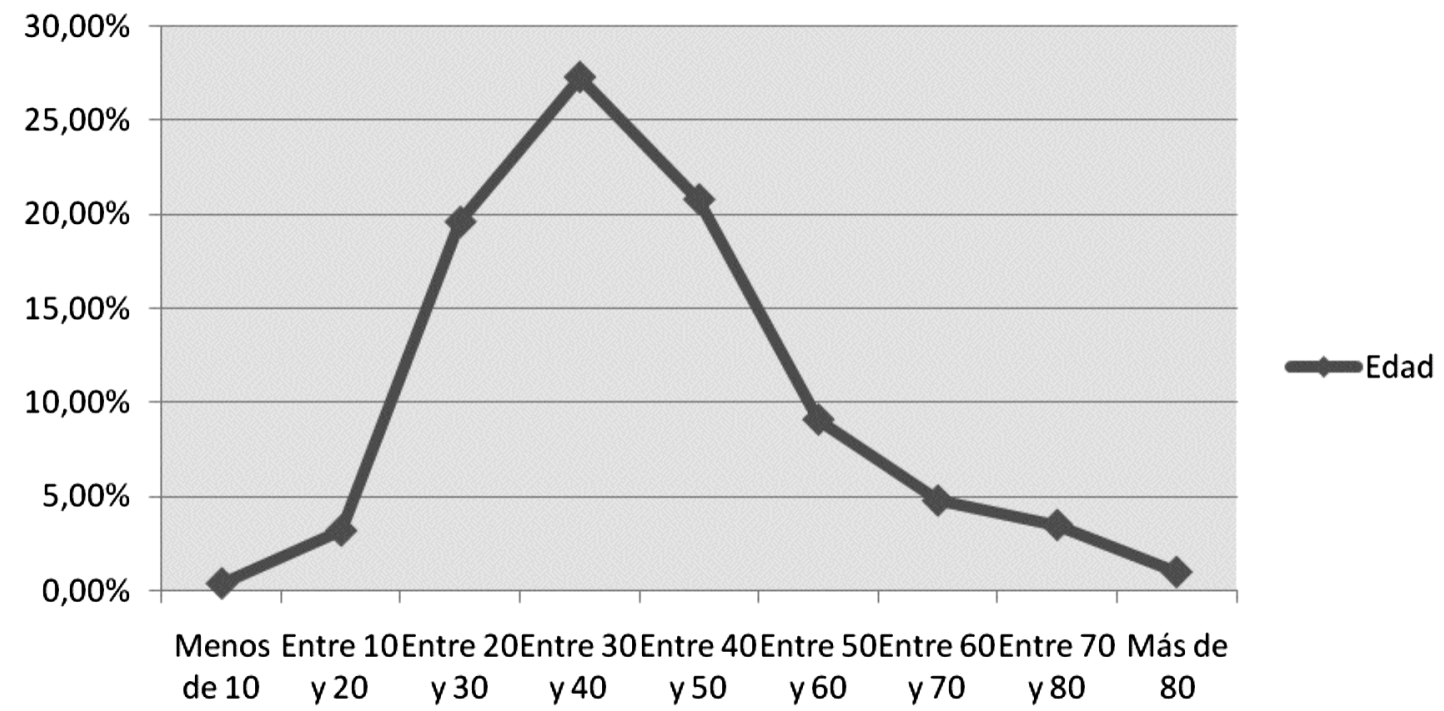

cabe duda es que la demora, esto es, el tiempo que transcurre entre que se comete un delito hasta que dicho delito es juzgado, es un indicador de eficacia. No suficiente, sin duda, pero sí necesario. Según nuestros datos, la demora media entre la comisión del delito y la fecha en la que se dicta la sentencia es

Tabla 5. Medidas de protección si hay convivencia

\begin{tabular}{lrr}
\hline Medidas de protección & Frecuencia & Porcentaje \\
\hline Salida domicilio convivencia y prohibición & & \\
$\quad$ volver & 84 & 3,3 \\
Intervención de armas & 47 & 1,9 \\
Internamiento psiquiátrico & 2 & 0,1 \\
Libertad provisional & 2 & 0,1 \\
Prohibición de acudir (art. 544 bis LECrim.) & 46 & 1,8 \\
Prohibición de aproximarse & 922 & 36,5 \\
Prohibición de comunicarse & 725 & 28,7 \\
Prisión provisional & 19 &, 8 \\
Prohibición de residir (ar. 544 bis LECrim.) & 58 & 2,3 \\
Otras medidas penales & 46 & 1,8 \\
Atribución del uso y disfrute de la vivienda & & \\
familiar & 139 & 5,5 \\
Régimen de custodia de los hijos & 142 & 5,6 \\
Régimen de estancia con los hijos & 7 &, 3 \\
Suspensión del régimen de visitas & 10 &, 4 \\
Régimen de comunicación con los hijos & 18 &, 7 \\
Régimen de prestación de alimentos & 135 & 5,4 \\
Régimen de visitas a los hijos & 99 & 3,9 \\
Permuta uso de la vivienda & 1 & 0,0 \\
Otras medidas civiles & 21 & 0,8 \\
\hline Total & 2523 & 100,0 \\
\hline
\end{tabular}

de 263 días (8,8 meses). La demora mínima es de un día y la máxima de 1719 días (casi 5 años). Sin embargo, al margen de estos extremos de carácter esencialmente anecdótico, debe tenerse en cuenta que el $25 \%$ de los casos se resuelve en menos de 10 días y que el $50 \%$ de los casos se resuelve en menos de 6 meses.

Por su parte, la demora media entre la comisión del delito y la fecha en la que dicha sentencia es firme, es de 317 días (10,6 meses). La demora mínima es de un día y la máxima de 2303 días (6,4 años). Sin embargo, como en el caso anterior, debe tenerse en cuenta que el $25 \%$ de los casos se resuelve en menos de 17 días y que el 50\% de los casos se resuelve en menos de 9,6 meses.

Para valorar esto resultados debemos plantearnos, por un lado, cuál es la demora que desde un punto de vista psicosocial y criminológico puede resultar óptimo. Por otro, qué expectativas realistas de logro se plantean los juzgados de violencia doméstica comparándolo, por ejemplo, con la actividad de otros juzgados del resto del Estado (no disponible en estos momentos) para, finalmente, carearlo con lo idealmente deseable: esto es, que los casos se resuelvan lo antes posible, sobre todo en aquellos casos que pueda estar comprometida la seguridad de las presuntas víctimas. 


\section{Reincidencia}

Una de las últimas variables de estudio que mejor pueden describir el perfil del agresor es la reincidencia. Ya hemos comentado que en este estudio, como en otros de similar naturaleza frecuentes en la literatura científica, se ha entendido la reincidencia como reiteración delictiva de un individuo que está implicado en dos o más causas, independientemente de la calificación penal de los delitos cometidos. En otros ámbitos de la Justicia, como la de Menores, la reincidencia es un indicador de eficacia del sistema de ejecución penal, ya que las sanciones son esencialmente concebidas como medidas educativas. Pero en el caso de la Violencia Doméstica, si bien existen programas de tratamiento para maltratadores, el objetivo de la Justicia, digámoslo claro, es fundamentalmente punitivo y disuasorio. Desde este punto de vista, y dada la gravedad de los delitos relacionados con la violencia doméstica, cabría esperar una baja reincidencia ya que, en principio, la privación de libertad restaría al infractor margen de error para cometer nuevos delitos. No es desde luego el caso. Debemos pensar, por tanto, que es tras la comisión de delitos leves o en aquellos que no hay privación de libertad o, en todo caso, tras haber cumplido dicha medida durante el periodo de estudio (2004-2007) que vamos a poder identificar los casos de reiteración delictiva.

En total, existen 4.948 infractores,- que hemos podido diferenciar gracias a un código de identificación personal,- de los cuales, 642 cometen dos o más delitos, lo que arroja una tasa de reincidencia del 13\%. Claro que, como se puede observar en la tabla 6 , la reincidencia puede consistir en haber cometido dos delitos o, como se aprecia en dos de los casos, hasta 8 delitos. En un periodo de tiempo como el

Tabla 6. Tasa de reincidencia de infractores

\begin{tabular}{|c|c|}
\hline $\begin{array}{c}N^{o} \text { de veces que } \\
\text { ha sido denunciado }\end{array}$ & $\begin{array}{l}N^{o} \text { de personas que han sido } \\
\text { denunciadas ese } n^{o} \text { de veces }\end{array}$ \\
\hline 2 & 483 \\
\hline 3 & 116 \\
\hline 4 & 25 \\
\hline 5 & 11 \\
\hline 6 & 5 \\
\hline 8 & 2 \\
\hline
\end{tabular}

estudiado cometer tres o más delitos puede considerarse una reincidencia muy alta.

Otra variable que también nos resultaba interesante describir es lo que vamos a denominar la reincidencia victimal. Lógicamente, si hay infractores reincidentes, es previsible encontrar víctimas que han cursado dos o más denuncias. Cabría profundizar el análisis intentando discernir si una misma persona es víctima de más de un individuo infractor, pero como ya adelantamos en el apartado al referirnos a la nacionalidad, dada la configuración actual de los datos, no es en este momento un análisis técnicamente viable. Por otra parte, podría entenderse que en una misma causa están implicados varios denunciantes o, por el contrario, también se puede plantear la hipótesis de que un mismo victimario puede llegar a atentar contra víctimas diferentes, pero no lo podemos confirmar.

\section{Conclusiones, discusión y líneas futuras de investigación}

- En casi un 58\% de las causas registradas no existe convivencia entre denunciado y denunciante.

- De todas las medidas registradas, el $80 \%$ corresponden a conflictos entre parejas, sean cónyuges, ex-cónyuges, novios, ex-novios u otras relaciones afectivas.

- Un 61,25\% de los delitos cometidos pueden ser considerados moderados ${ }^{1}$. El porcentaje de delitos graves ${ }^{2}$ llega al 20\%. El balance global

\footnotetext{
${ }^{1}$ Moderado: lesiones no significativas en el contexto familiar, injerencias significativas en la integridad corporal o salud psicofísica por imprudencia grave, participación en un contexto idóneo para afectar a la vida o causar significativas afecciones de la integridad corporal o la salud psicofísica, involucrar a otra persona en un contexto sexual no voluntario, omitir la ayuda en una situación de peligro para bienes personales de otro, invadir la privacidad ajena en un contexto tan sensible para la percepción de intimidad y seguridad personal como el domicilio, afectar a la dignidad ajena con expresiones o palabras, emplear medios específicos para quebrar el marco de custodia del patrimonio ajeno, causar desperfectos relevantes en el patrimonio ajeno de forma intencionada, producir daños colectivos de forma imprudente, crear un riesgo específico para la vida y salud ajena en un contexto de riesgo significativo como la circulación de vehículos a motor y afectar de forma relevante a los intereses públicos o institucionales.

${ }^{2}$ Grave: injerencias relevantes en la integridad corporal o salud psicofísica, creación de un espacio de dominio psicofísico en el contexto familiar, afectaciones de la libertad personal, lesión de la libertad sexual, empleo de la violencia o la intimidación para lesionar el patrimonio ajeno.
} 
apunta a una gravedad acusada en los delitos relacionados con la violencia intrafamiliar.

- A partir del año 2004, se incrementa prácticamente un $100 \%$ el volumen de sentencias dictadas en los juzgados de violencia doméstica, manteniéndose una tendencia estable durante el trienio siguiente (2005-2007).

- Un 23,2\% de los imputados son extranjeros entre los que destaca el colectivo latino-americano. En el caso de los y las denunciantes nos encontramos con proporciones comparables.

- Un 27,35 de los y las denunciantes son personas entre 30 y 40 años.

- Las medidas cautelares más habituales, si hay convivencia, son la prohibición de aproximarse $(36,5 \%)$ y la prohibición de comunicarse $(28,7 \%)$.

- Aproximadamente un $13 \%$ de los imputados vuelve a reincidir en el periodo de estudio.

- La demora media entre la comisión del delito y cuando se dicta la sentencia es de 263 días $(8,8$ meses). El $25 \%$ de los casos se resuelve en menos de 10 días y el $50 \%$ de los casos se resuelve en menos de 6 meses.

\section{Discusión}

\section{La conciencia social}

Teniendo en cuenta lo que hemos comentado sobre la interpretación de las estadísticas, cabe decir que el informe presentado recientemente por la ministra de Igualdad, Bibiana Aído, es considerado por ella misma como una evidencia de que el grado de sensibilización de la sociedad hacia el maltrato y el asesinato de mujeres es mínimo, y que la puesta en marcha de la ley y las campañas de concienciación realizadas por todas las instituciones públicas han servido de poco. Así lo ponen de manifiesto los distintos barómetros del Centro de Investigaciones Sociológicas (CIS). La violencia doméstica aparece como uno de los problemas más importantes para los españoles en el año 2000. En ese momento, un $2,7 \%$ de los entrevistados aludió a la violencia machista como uno de los tres problemas más graves de España. El grado más alto de concienciación llegó en el año 2004, mientras se debatía la Ley integral de Violencia de Género que entró en vigor el año siguiente. La preocupación por este asunto fue mencionada entonces por el $6,7 \%$ de los encuestados. Pero desde entonces ha vuelto a descender, hasta el 2,7\% registrado el año pasado, cuando precisamente en nuestro estudio se incrementa en casi un $100 \%$ el volumen de sentencias dictadas.

De hecho, en el último barómetro publicado en abril, este problema se sitúa como el undécimo en relevancia, a enorme distancia de los cinco primeros. El paro fue mencionado por el $52 \%$ de los encuestados y los problemas económicos por el $48,2 \%$, seguidos por el terrorismo (31\%), la inmigración (26\%) y la vivienda (25\%). Conclusión preliminar: algo no estamos haciendo bien atribuyendo a la sensibilización contra el inefable machismo toda la respuesta a la solución del problema.

\section{Un problema grave y complejo}

Sin embargo, el "feminismo oficial" ha elevado a corrección política un panmachismo en virtud del cual existe un delito que solo puede ser cometido por hombres para los que se reservan una serie de medidas que, según el foro de debate, pueden ir desde las penas de prisión hasta la castración física y/o química, pasando por la publicación de listas de maltratadores independientemente que hayan cumplido ya su castigo y, todas ellas, bajo el paraguas del manido "tolerancia cero" de la campaña institucional diseñada al efecto (Redondo, 2009). En la línea de otros trabajos fuera de España (Straus, 2008 o Williams et al, 2008), el discurso marco de estas iniciativas, según Elena Larrauri (2007), presentaría cuatro características distintivas: “... 1) Simplifica en exceso la explicación, atribuyendo el maltrato a la mujer al propio hecho de ser mujer; 2) Segrega este problema de violencia como algo aparte y distinto del resto de los comportamientos violentos; 3 ) Para realzar la desigualdad de género, ignora esencialmente otras desigualdades sociales, de indole económica, social, etc. y 4) Confía al derecho penal la tarea de erradicar la desigualdad/violencia de género". 
En un estudio realizado por el Instituto de la Mujer en el año 2006 con una muestra de 32.000 mujeres, se constata que en España hay al menos, un $3,6 \%$ de mujeres mayores de 18 años que son maltratadas por su pareja (alrededor de 680.000). Sin embargo, hay un $6 \%$ adicional que aun no considerándose maltratadas, sufren conductas vejatorias que son impropias en una relación de pareja sana. A partir de este dato y si se diera, por otra parte, una sobreutilización del sistema judicial en casos de fracaso en la relación de pareja, nos encontraríamos con un porcentaje indeterminado de mujeres que podrían estar presentando denuncias sobrevaloradas basadas en conflictos domésticos no resueltos, además de ese $6 \%$ de mujeres víctimas de comportamientos impropios en el seno de una relación de pareja que, por diferentes motivos, no solicitan ayuda, no ya judicial, sino tampoco de tipo psicosocial. Por otra parte, en el ámbito de los problemas de violencia intrafamiliar, es evidente la muy deficitaria visibilidad del maltrato infantil y no digamos del creciente fenómeno de la violencia contra las personas mayores. Es decir, todo parece indicar que ni están todos los que son, ni son todos los que están, por expresarlo de una forma poco técnica.

En todo caso, seguimos enfrentándonos a un porcentaje alarmante de mujeres víctimas de la violencia machista, lo que ha suscitado en la comunidad científica un aumento del interés por el estudio de este fenómeno y en concreto de los agresores (Romero, Rodriguez \& Echauri, 2005). Según FernándezMontalvo \& Echeburúa (2008), éstos suelen presentar carencias psicológicas significativas, tales como sesgos cognitivos (pensamientos distorsionados sobre los roles sexuales y la inferioridad de la mujer y sobre la justificación de la violencia), dificultades de comunicación y de resolución de problemas, irritabilidad y una falta de control de los impulsos, así como otras dificultades específicas como pueden ser los celos patológicos. Seguir investigando en esta línea puede ser un objetivo prioritario.

\section{La eficacia de la ley}

El proceso de implementación de la Ley integral contra la violencia de género puede contribuir, y para eso ha sido concebida, a una mayor protección y mejora de la asistencia a la víctima y a una mayor sensibilización de la población. Sin embargo existen autores que alertan sobre sus posibles efectos criminalizadores (Camps \& Schmal, 2007). Desde nuestro punto de vista, creemos necesario mejorar los procedimientos y protocolos de recogida de datos por parte del Departamento de Justicia, con el fin de poder diseñar investigaciones rigurosas y fiables orientadas a contrastar la eficacia de las medidas sancionadoras, por un lado, y conocer en mayor detalle, como ya hemos mencionado, los perfiles psicosociales de víctima y victimario, por otro. En este sentido sería interesante, por ejemplo, analizar las circunstancias que rodean a las víctimas que retiran la denuncia. Tengamos en cuenta que el diez por ciento de las denuncias presentadas por violencia machista acaban en nada porque una de cada diez querellantes renuncia al proceso, según los datos facilitados por el Observatorio contra la Violencia Doméstica y de Género. Este porcentaje llega al $12,4 \%$ en el caso del País Vasco (tabla 7).

En este punto es oportuna la cita que trae a colación Redondo (2009) de Quintero Olivares “...La clase política española ha propiciado la idea de que cualquier problema ha de encontrar remedio en los cambios legales, incluyendo en cabeza, los cambios de las leyes penales, lo que implica atribuir la "causa" del problema a insuficiencia de las leyes. Se añade una inquebrantable fe en la relación entre terapéutica legislativa y desaparición del mal; pero situar las

Tabla 7. Tasa denuncias-Renuncias-Órdenes de protección (Primer trimestre de 2008)

\begin{tabular}{lcccccc}
\hline & $\begin{array}{c}\text { Denuncias } \\
\text { Recibidas }\end{array}$ & $\begin{array}{c}\text { Denuncias por } \\
\text { cada 10.000 hab. }\end{array}$ & $\begin{array}{c}\text { Denuncias por } \\
\text { cada 10.000 mujeres }\end{array}$ & $\begin{array}{c}\text { Renuncias } \\
\text { al proceso }\end{array}$ & $\begin{array}{c}\text { Ratio renuncias } \\
\text { denuncias }\end{array}$ & $\begin{array}{c}\text { Ratio órdenes/ } \\
\text { denuncias }\end{array}$ \\
\hline España & 33.950 & 7,5 & 14,6 & 3.322 & $9,8 \%$ & $28 \%$ \\
País Vasco & 1.007 & 4,7 & 9,2 & 125 & $12,4 \%$ & $28 \%$ \\
\hline
\end{tabular}

Fuente: Consejo General del poder Judicial. Elaboración propia 
leyes en el centro de los problemas es un error que se paga pronto, pues la perseverancia del problema provoca inevitable sensación de impotencia"

\section{Mujer inmigrante víctima}

Un mensaje recurrente en los medios de comunicación, campañas de sensibilización y referencias especializadas es que la violencia intrafamiliar, en general, y la violencia contra la mujer, en particular, "afecta a todas las capas y estratos sociales". Es cierto. Pero no es menos cierto que en torno al $20 \%$ de las sentencias registradas judicialmente están relacionadas con víctimas extranjeras, y se nos antoja que no precisamente de "todas" las clases sociales. En el informe anteriormente citado presentado ante el Consejo de Ministros se expone cómo la evolución del porcentaje de mujeres extranjeras asesinadas ha pasado del $20 \%$ en el 2004 a casi el $40 \%$ el año pasado.

Lo que queremos decir es que la corrección política del discurso no nos sirva de coartada para obviar que existen factores de riesgo que hacen a determinados colectivos objetivamente más vulnerables. Porque los hay, como puede ser el caso de la mujer inmigrante.

Este y los demás aspectos señalados pueden reescribirse en forma de retos. Retos de la investigación aplicada a la comprensión y prevención de la violencia intrafamiliar. Retos, en fin, con los que tenemos, debemos y queremos comprometernos.

\section{Referencias}

Ballonga, J., Cánovas, C., Casany, R., Caso, C., Gimeno, J., Guillamat, A., Juliá, M., Mercadal, C., Ortí, M., Sala, M. y Valbona, E. (2007). Estudio descriptivo de familias con dinámicas de violencia doméstica en un contexto judicial de orden civil. Anuario de Psicología Jurídica, 17, 9-41.

Centro Reina Sofía: http://www.centroreinasofia.es/ Consejo General del Poder Judicial: Datos de denuncias, procedimientos penales y civiles registrados, órdenes de protección solicitadas en los juzgados de violencia sobre la Mujer y sentencias dictadas en el primer trimestre del año 2008.
http://www.poderjudicial.es/eversuite/GetRecord s?Template $=$ cgpj/cgpj/principal.htm (Recuperado en abril de 2009).

Consejo General del Poder Judicial. Grupo de Expertos/as en Violencia Doméstica y de Género del CGPJ. Análisis de las sentencias dictadas por los tribunales del jurado en el periodo (20012005), relativas a homicidios y asesinatos entre los miembros de la pareja o ex pareja. Conclusiones. http://www.poderjudicial.es/eversuite/Get Records ? Template $=\mathrm{cgpj} / \mathrm{cgpj} /$ principal.htm (Recuperado en abril de 2009).

Cuesta, J.L. de la (2006). El maltrato de personas mayores: Detección y prevención desde un prisma criminológico interdisciplinar. San Sebastián: Fundación Hurkoa-Instituto Vasco de Criminología.

Fernández-Montalvo, J. y Echeburúa, E. (2008): Violencia de pareja: Perfil psicológico del agresor y programas de intervención. Infocop, 38, pp. 6-8

Instituto de la Mujer: http://www.migualdad.es/ mujer/ (Recuperado en abril de 2009).

Ley Orgánica1/2004, de 28 de diciembre, de Medidas de Protección Integral contra la Violencia de Género.

Ministerio del Interior: http://www.mir.es/ (Recuperado en abril de 2009).

Observatorio Vasco de Inmigración: http://www. violenciaintrafamiliar.org/espana/portada.php (Recuperado en abril de 2009).

Redondo, S. (2009). In-Tolerancia Cero: Un mundo con menos normas, controles y sanciones también sería posible. Barcelona: Sello Editorial.

Romero, J., Rodriguez, M.J. \& Echauri, J.A. (2005). Teoría y descripción de la violencia doméstica: Programa terapéutico para maltratadores del ámbito familiar en el centro penitenciario de Pamplona. Anuario de Psicología Jurídica, 15, 67-91.

Straus, M.A. (2008). Bucking the tide in family violence research. Trauma, Violence \& Abuse, 9, 191-213.

Williams, J.R., Ghandour, R.M. y Kub, J.E. (2008). Female perpetration of violence in heterosexual intimate relationships: Adolescence to Adulthood. Trauma, Violence \& Abuse, 9, 227-249.

Manuscrito recibido: 13/04/2010

Revisión recibida: 19/04/2010

Manuscrito aceptado: 26/04/2010 